\title{
Mecanismos Explicativos das Falsas Memórias no Paradigma DRM
}

\section{Explanatory Mechanisms of False Memories in DRM Paradigm}

\author{
Helena Mendes Oliveira ${ }^{a, b}$ \& Pedro Barbas de Albuquerque*, \\ ${ }^{a}$ Instituto Superior da Maia, Maia, Portugal \& ${ }^{b}$ Universidade do Minho, Braga, Portugal
}

\begin{abstract}
Resumo
Neste artigo apresenta-se uma revisão da literatura sobre as principais teorias e mecanismos explicativos da ocorrência de falsas memórias no paradigma Deese-Roediger-McDermott (DRM). São também apresentados dados de artigos empíricos que suportam cada uma das teorias, e discutidas as suas implicações para a compreensão deste fenômeno. Historicamente, as primeiras explicações teóricas centravam-se no conceito de associação para explicar as intrusões em tarefas de memória. Mais tarde, o conceito de ativação assumiu grande relevância para explicar a ocorrência de memórias falsas no paradigma DRM. Contudo, o conceito de ativação revelou-se insuficiente para explicar alguns resultados descritos por vários autores. Assim, junto com os mecanismos de ativação, as duas grandes teorias explicativas (teoria de ativação e monitoramento e teoria do traço difuso) consideram a existência de mecanismos de monitoramento ou controle. Os mecanismos de monitoramento permitem explicar a diminuição ou o desaparecimento das memórias falsas em algumas condições experimentais. Apesar de serem diferentes, as visões de ambas as teorias não são inconciliáveis e talvez sejam necessárias para a integral compreensão das falsas memórias no paradigma DRM. Assim, propõe-se a adoção de uma abordagem teórica que integre contributos das duas teorias.

Palavras-chave: Falsas memórias, DRM, teoria do traço difuso, teoria de ativação e monitoramento, teoria do monitoramento da fonte.
\end{abstract}

\begin{abstract}
This literature review explores the major theories and explanatory mechanisms in the occurrence of false memories in the Deese-Roediger-McDermott paradigm (DRM). This study presents data from empirical studies that support each of the theories and discusses their implications for understanding false memories. Historically, the first theories explaining false memories focused on the concept of association in order to explain intrusions in memory tasks. Later on, the concept of activation received greater importance in explaining false memories in the DRM paradigm. However, activation by itself is insufficient to explain all the results described by several authors. Therefore, the two current major theories that explain false memories in the DRM paradigm (activation monitoring theory and fuzzy trace theory) consider the existence of monitoring or control mechanisms, together with activation mechanisms. These monitoring mechanisms, which operate in opposite direction to activation mechanisms, explain the decrease or disappearance of false memories under certain circumstances. Although different, activation monitoring and fuzzy trace theories have reconcilable perspectives and may be both necessary for a comprehensive understanding of false memories in the DRM paradigm. Thus, we propose the adoption of a theoretical approach that integrates contributionsfrom both theories.

Keywords: False memories, DRM, fuzzy trace theory, activation monitoring theory, source monitoring.
\end{abstract}

Ao longo de mais de um século de estudo das falsas memórias, foram várias as explicações e os mecanismos propostos com o objetivo de clarificar o motivo pelo qual, numa dada circunstância, uma pessoa é levada a recordar ou reconhecer um evento novo como tendo ocorrido anteriormente, ou a fazê-lo de forma distorcida. No caso do paradigma Deese-Roediger-McDermott (DRM), o procedi-

\footnotetext{
* Endereço para correspondência: Universidade do Minho, Escola de Psicologia, Campus de Gualtar, Braga, Portugal 4710-057. Fones: 0035 1253604220, 0035 1253604683; Fax: 0035 1253604224. E-mail: helena. mendes.oliveira@gmail.com e pedro.b.albuquerque@psi.uminho.pt
}

mento consiste na apresentação de listas de palavras (e.g., mesa, sentar, pernas, assento, macio, secretária, braço, sofá, madeira, almofada, descanso e banco), todas elas associadas a uma outra palavra não apresentada (e.g., cadeira), comummente designada por distrator crítico. Após a apresentação destas listas seguem-se tarefas de recordação e/ou de reconhecimento, sendo que o distrator crítico tende a ser recordado e reconhecido na mesma proporção que as palavras apresentadas nas listas (Deese, 1959b; Roediger \& McDermott, 1995; Stein, Feix, \& Rohenkohl, 2006; Stein \& Pergher, 2001; Toglia, Neuschatz, \& Goodwin, 1999).

Neste trabalho, refletiremos sobre os mecanismos 
explicativos que têm sido apontados como estando na origem do surgimento de falsas memórias no paradigma DRM, tendo por base as duas teorias que, atualmente, são mais comummente usadas para explicar este fenômeno: a teoria de ativação e monitoramento (Gallo \& Roediger, 2002; Roediger, Balota, \& Watson, 2001; Roediger \& McDermott, 1995, 2000a, 2000b) e a teoria do traço difuso (Brainerd \& Reyna, 2005; Reyna \& Brainerd, 1995). Para tal, apresentaremos uma revisão de artigos científicos que recorreram ao procedimento DRM para estudar as falsas memórias e discutiremos as suas implicações à luz de cada uma das teorias.

Deese (1959b) foi pioneiro ao aplicar este procedimento ao estudo das falsas memórias verificando uma taxa de recordação do distrator crítico que variou entre $0 \%$ (lista borboleta) e 44\% (lista sono). Em 1995, Roediger e McDermott (1995) replicaram o estudo de Deese (1959b), utilizando as seis listas que tinham gerado as taxas de recordação falsa mais elevadas e constataram que o distrator crítico foi recordado, em média, em $40 \%$ dos casos, um valor semelhante ao obtido na recordação das palavras apresentadas no meio das listas. Além da tarefa de recordação, os participantes realizaram tarefas de reconhecimento. Nesta tarefa não se verificaram diferenças entre os valores obtidos no reconhecimento das palavras apresentadas (86\%) e para as falsas memórias dos distratores críticos (84\%). Com este padrão geral de resultados ficou, assim, estabelecido que a produção de falsas memórias em contexto laboratorial é possível e pode mesmo assumir valores elevados, quando comparados com a recordação de itens efetivamente apresentados aos participantes.

Tendo sido o primeiro a estudar esta forma de produção de falsas memórias, compreende-se que a tentativa inicial de enquadrar teoricamente este fenômeno esteja presente nos trabalhos de Deese (1959a, 1959b). Este autor recorreu então ao conceito de associação para explicar a elevada taxa de intrusões para o distrator crítico, encontrada em algumas das suas listas de palavras. Assim, o distrator crítico seria tanto mais ativado pela apresentação das palavras que the estivessem associadas, quanto mais forte fosse essa associação. E a intensidade da força associativa das palavras apresentadas ao distrator crítico seria um bom preditor da sua recordação ou reconhecimento posterior (ou seja, produção de falsa memória).

Esta não foi a primeira vez que o conceito de associação foi usado para explicar a ocorrência de erros de memória deste tipo. Mais de 60 anos antes, Kirkpatrick (1894) recorreu ao conceito de associação para explicar a ocorrência de algumas "ilustrações incidentais de falsa recordação" (p. 608). Este autor afirmou que algumas palavras não apresentadas eram recordadas pelo fato de estarem "frequentemente associadas" (p. 608) a outras palavras apresentadas. Antes disso, já Ebbinghaus (1885/1964), na primeira aplicação do método experimental ao estudo da memória humana, havia detetado o surgimento de falsas memórias em listas de sílabas sem sentido (e.g., BOL, DAX, YAT), demonstrando a possibilidade das associa- ções se formarem sobre material novo, sem nenhum tipo de representação mental prévia.

Decorridos poucos anos após os trabalhos de Deese (1959a, 1959b), que demonstram claramente a ocorrência de recordação falsa com listas de palavras, e na sequência da utilização do mesmo tipo de material para estudar o fenômeno em tarefas de reconhecimento, surgiu um primeiro modelo explicativo destes resultados, que ficou conhecido como a teoria da ativação implícita (Underwood, 1965). De acordo com esta teoria, a apresentação de um estímulo produziria a ativação da representação mental desse estímulo e, também, a ativação da representação mental de estímulos associados (resposta associativa implícita). Segundo Underwood, esta ativação seria não intencional, mas consciente, e surgiria, no caso das listas semanticamente associadas, no momento da apresentação das palavras da lista. Assim, e de acordo com este pressuposto, na fase de teste, as palavras não apresentadas, mas semanticamente associadas, tenderiam a ser reconhecidas como se tivessem sido apresentadas. Na verdade, vários estudos (Brainerd \& Reyna, 1998; Pimentel \& Albuquerque, 2013; Roediger $\&$ McDermott, 1995; Stein et al., 2006; Stein \& Pergher, 2001; Toglia et al., 1999) mostram que, ao nível do reconhecimento, não existem diferenças entre a porcentagem de êxitos e a de falsos alarmes para o distrator crítico, nem no tipo de julgamentos efetuados (e.g., lembro/sei - de acordo com a terminologia de Tulving, 1985), e, em tarefas de recordação, o distrator crítico iguala, ou ultrapassa mesmo, a taxa de recordação de algumas palavras apresentadas nas posições intermediárias das listas. Estes resultados parecem suportar a visão de que o distrator crítico é, de fato, ativado na fase de estudo. Mais tarde, outros autores propuseram que a resposta associativa implícita poderia surgir tanto na fase de estudo como na fase de teste, no momento da recordação e reconhecimento das palavras apresentadas (Cramer, 1970).

Embora as explicações variem, o conceito de associação encontra-se bem patente nestas primeiras tentativas de enquadramento teórico das falsas memórias produzidas a partir da apresentação de listas de palavras. Tanto Deese (1959a, 1959b), como Underwood (1965), baseiam as suas explicações no pressuposto de que a organização da informação na memória obedece a princípios associativos. No entanto, nenhum deles esclarece exatamente o processo através do qual a apresentação de um conjunto de palavras associadas desencadeia a recordação e o reconhecimento de outras.

$\mathrm{Na}$ verdade, o conceito de associação aplicado à forma como a memória humana se organizava, permaneceu vago até às décadas de 1960 e 1970, período em que vários experimentos procuraram testar empiricamente o modo como determinados conceitos estavam efetivamente associados a outros na memória. Este conjunto de experimentos foi conduzido por Collins e Quillian (1969, 1970), depois de Quillian (1967) ter proposto um modelo computacional da estrutura e do funcionamento da memória semântica. De acordo com este modelo, com cada conceito é armazenado 
na memória um conjunto de ligações a outros conceitos, sendo que a configuração de ligações de uma palavra representa o seu significado. Trata-se de um modelo hierárquico vertical, em que a informação relativa a um conceito de ordem superior se generaliza aos conceitos de ordem inferior, por exemplo, se o conceito de "pássaro" inclui propriendades como "tem penas" e "voa", então, sendo um canário também um pássaro, automaticamente se assume que um canário tem penas e voa.

Durante este período, realizaram-se outros experimentos tendo em vista a compreensão da forma como a memória semântica se organizava (Rips, Shoben, \& Smith, 1973), e surgiram outros modelos explicativos do funcionamento da memória (E. E. Smith, Shoben, \& Rips, 1974). Com base nestes experimentos e modelos teóricos, Collins e Loftus (1975) propuseram uma teoria que, segundo os próprios autores, seria uma extensão da teoria apresentada por Quillian (1967), e que viria a assumir um papel de destaque na compreensão do funcionamento da memória semântica, e a servir de base a muitas das teorias que se lhe seguiram, como a teoria da propagação da ativação.

A ideia-chave desta teoria é a de que, quando se dá o processamento de um estímulo, há uma ativação do nó (node) correspondente à representação mental desse mesmo estímulo e que essa ativação se espalha aos nós mais próximos, que correspondem a representações mentais de conceitos que têm alguma relação com o estímulo inicial. Esta ativação será tanto mais forte e duradoura, quanto maior for a proximidade (e.g., semântica, fonológica, perceptiva) dos nós em questão. Este modelo também assenta no pressuposto da organização associativa da memória, ou seja, é pelo fato da memória estar organizada em rede, sendo composta por nós que estão ligados entre si, que, mediante a apresentação de um determinado estímulo que gera a formação de um traço de memória, outros estímulos que se lhe são próximos sofrem também alguma forma de ativação (Collins \& Loftus, 1975).

Assim, no caso particular das falsas memórias no procedimento DRM, tratando-se de uma tarefa em que se apresenta um conjunto de palavras associadas a uma outra não apresentada (distrator crítico), a posterior recordação e reconhecimento do distrator crítico seria resultado da ativação repetida do nó correspondente à sua representação mental, na sequência da apresentação dos seus associados. Dessa maneira, a convergência associativa das palavras de uma lista explicaria, em boa parte, as elevadas taxas de recordação e reconhecimento falsos registrados na maioria dos estudos realizados (Meade, Watson, Balota, \& Roediger, 2007; Roediger, Balota, et al., 2001).

Apesar da teoria da propagação da ativação defender que seriam as relações semânticas que determinariam a proximidade entre as representações mentais dos conceitos, no caso do paradigma DRM, o conceito de associação não se circunscreve a este tipo de relações, assumindo-se, através da determinação da força associativa entre as palavras de uma lista e o distrator crítico, a quantificação de um critério operativo. Em outras palavras, a associação entre duas palavras é tão mais forte, quanto maior for a probabilidade de uma (alvo) gerar a outra (tema) em tarefas de associação livre. Ainda assim, análises efetuadas à natureza das relações entre as palavras das listas habitualmente usadas neste paradigma e os respectivos distratores críticos, apontam para um forte componente semântico dessas relações (Brainerd, Yang, Reyna, Howe, \& Mills, 2008; Cann, McRae, \& Katz, 2010; McRae, Khalkhali, \& Hare, 2012).

A teoria da propagação da ativação permitiu compreender melhor os dados anteriormente obtidos por Deese (1959a, 1959b), Kirkpatrick (1894) e Underwood (1965). Os resultados apresentados por estes autores ajustam-se bem a este modelo, que explica, por exemplo, porque é que o aumento da força associativa tem como resultado o aumento das falsas memórias. No entanto, já não explica tão bem porque é que existe uma correlação negativa entre recordação falsa e correta, um dos resultados reportados por Deese (1959a, 1959b), e confirmado por muitos estudos posteriores (Roediger \& McDermott, 1995; Roediger, Watson, McDermott, \& Gallo, 2001). Se houvesse uma relação linear entre a ativação do distrator crítico e a sua posterior recordação ou reconhecimento, então seria de esperar que quanto mais palavras os participantes conseguissem recordar e reconhecer, mais forte fosse a ativação do distrator crítico e, assim, maior fosse a probabilidade de aparecimento da falsa memória. Porém, isso não acontece, o que faz supor que outros mecanismos estão envolvidos neste processo.

Aliás, rapidamente se percebeu que este fenômeno não poderia ser explicado apenas pela via da ativação. Apesar das elevadas taxas de recordação falsa encontradas por Deese (1959b), havia listas em que o fenômeno não ocorria, não obstante os elevados valores de força associativa das palavras que compunham estas listas em relação ao distrator crítico. Portanto, se a ativação e consequente produção de falsas memórias pudesse ser explicada, sobretudo, por via da força associativa das palavras apresentadas com o distrator crítico, como se justificaria que listas com forças associativas semelhantes pudessem não gerar falsas memórias?

Após terem replicado o estudo de Deese (1959b) recorrendo a uma tarefa de recordação livre, e terem demonstrado a ocorrência de um fenômeno semelhante em tarefas de reconhecimento, Roediger e McDermott (1995) também apelaram aos mecanismos de ativação para dar conta dos seus resultados. No entanto, fizeram-no assumindo o papel que os mecanismos de controle ou monitoramento desempenhariam no fenômeno. Em particular, os autores aludiram à teoria do monitoramento da fonte, proposta por Johnson, Hashtroudi e Lindsay (1993) para explicar o fato dos participantes afirmarem lembrar-se de ter ouvido o distrator crítico ao invés de referirem apenas que sabiam que este havia sido apresentado. De acordo com Roediger e McDermott (1995), durante a tarefa de reconhecimento, os participantes confundiam a fonte da informação, julgando erradamente que o distrator crítico 
havia estado presente nas listas, quando, na verdade, ele apenas teria sido ativado durante a fase de apresentação das palavras associadas, graças a um fenômeno de ativação por contágio (Collins \& Loftus, 1975).

Assim, além dos mecanismos de ativação, as abordagens explicativas deste fenômeno contemplam, também, mecanismos de controle ou de monitoramento. Atualmente, e como já referimos, existem duas grandes teorias explicativas das falsas memórias produzidas a partir da apresentação de listas de palavras associadas: a teoria de ativação e monitoramento, proposta por Roediger e colaboradores (Gallo \& Roediger, 2002; Roediger, Balota, et al., 2001; Roediger \& McDermott, 1995, 2000a, 2000b; Roediger, Watson, et al., 2001), e a teoria do traço difuso (Brainerd \& Reyna, 2005; Reyna \& Brainerd, 1995). Quer uma, quer outra, preveem a existência de mecanismos que operam em direções contrárias e que pretendem explicar os motivos pelos quais as falsas memórias surgem, assim como as razões que levam à sua diminuição ou mesmo desaparecimento. Os primeiros são comumente designados de mecanismos de ativação e permitem explicar o porquê de, em algumas situações, os participantes referirem o distrator crítico como tendo sido apresentado. Por outro lado, os mecanismos de monitoramento ou controle permitem compreender porque motivo não se dá a recordação e o reconhecimento do distrator crítico em outras situações. Vamos procurar realizar uma apresentação mais detalhada destes mecanismos a seguir.

\section{Mecanismos de Ativação}

Entende-se por mecanismos de ativação os processos através dos quais um estímulo é ativado em consequência da apresentação de um outro estímulo que tem com ele algum tipo de associação. Essa ativação pode resultar na sua recordação ou reconhecimento posterior pelos participantes de um experimento, como se de um estímulo apresentado se tratasse (Gallo, 2010). À luz das teorias que a seguir apresentaremos, a ativação da representação mental de um conceito é uma condição tida como necessária para que surja uma falsa memória.

A teoria de ativação e monitoramento foi proposta por Roediger e colaboradores (Gallo \& Roediger, 2002; Roediger, Balota, et al., 2001; Roediger \& McDermott, 1995, 2000a, 2000b; Roediger, Watson, et al., 2001) para enquadrar do ponto de vista teórico o aparecimento de falsas memórias no paradigma DRM. Esta teoria assenta em outros modelos teóricos anteriormente desenvolvidos para explicar parcialmente, quer a recordação e reconhecimento corretos, quer as falsas memórias. Entre estes modelos destacam-se a teoria da resposta associativa implícita, proposta por Underwood (1965); a teoria da propagação da ativação (Collins \& Loftus, 1975); e a teoria do monitoramento da fonte (Johnson et al., 1993). Enquanto as duas primeiras constituem tentativas de explicar os mecanismos que favorecem o aparecimento das falsas memórias, a teoria do monitoramento da fonte permite explicar os mecanismos que impedem o seu aparecimento ou levam à sua inibição.

À luz da teoria de ativação e monitoramento, a apresentação de um conjunto de palavras de uma lista gera a ativação de palavras associadas, nomeadamente do distrator crítico, que, por definição, é uma palavra com a qual todas as palavras apresentadas estão relacionadas. Então, quando uma palavra é apresentada, dá-se a ativação do nó correspondente à representação mental desse conceito, e, devido a um fenômeno de propagação da ativação, os nós que no léxico dos participantes têm com aquele algum tipo de relação são também ativados (Roediger, Balota, et al., 2001). A ativação do distrator crítico pode ocorrer tanto na fase de estudo, como na fase de teste, durante a recordação das palavras apresentadas ou em tarefas de reconhecimento (Meade et al., 2007).

Segundo a teoria do traço difuso (Brainerd \& Reyna, 2005; Reyna \& Brainerd, 1995), no momento da codificação dá-se a formação de dois tipos de traços de memória, um traço de essência e um traço literal. O traço literal comporta a codificação da informação específica dos estímulos, como as suas características perceptivas, enquanto o traço de essência diz respeito à codificação de aspectos mais amplos e inespecíficos, como a interpretação do seu significado (gist). Assumindo a perspectiva da teoria do traço difuso, a apresentação de um conjunto de palavras associadas a uma outra não apresentada desencadeia, junto com a codificação das características específicas de cada uma das palavras apresentadas, a formação de um gist, ou tema central da lista. Este tema central, que é extraído e codificado no momento da apresentação das palavras da lista, será um tema aglutinador que, em muitos casos, coincidirá com o distrator crítico dessa mesma lista. A sua extração facilita a recordação e reconhecimento das palavras apresentadas, mas também a de outras com elas relacionadas, nomeadamente do distrator crítico, caso este coincida com o tema identificado pelos participantes (Brainerd \& Reyna, 2005). A aceitação do distrator crítico como se fosse uma palavra das listas, ocorrerá, de acordo com esta teoria, devido a um processo de julgamento de similaridade, na medida em que as características semânticas do distrator crítico são comuns às das palavras apresentadas. No entanto, os autores não rejeitam a possibilidade de que a recordação do distrator crítico possa ser atribuída, em alguns casos, a uma recordação fantasma (existência de uma "memória" vívida para um evento que não ocorreu), e não apenas à sensação de familiaridade provocada pela partilha de características entre o distrator crítico e as palavras apresentadas (Brainerd, Wright, Reyna, \& Mojardin, 2001).

Embora a teoria de ativação e monitoramento e a teoria do traço difuso não apresentem visões inconciliáveis no que diz respeito aos mecanismos de ativação, há alguns aspectos que as distinguem, designadamente a natureza das relações associativas (e.g., semântica, fonológica, situacional, categorial) entre as palavras apresentadas e o distrator crítico da lista. Enquanto a teoria de ativação e monitoramento faz referência às associações entre as 
palavras (sem especificar a natureza dessas associações), defendendo que a força associativa reversa é a principal variável explicativa do aparecimento de falsas memórias neste paradigma, a teoria do traço difuso postula que a ocorrência deste fenômeno se deve, sobretudo, ao fato das palavras apresentadas terem, entre si, e com o distrator crítico, algum tipo de relação semântica, que conduz, na maior parte dos casos, à extração do significado global da lista (gist ou tema da lista) e à sua posterior recordação e reconhecimento (Brainerd \& Reyna, 2005; McRae et al., 2012).

Tendo em conta o vasto conjunto de estudos realizados sobre falsas memórias recorrendo ao paradigma DRM (Brainerd \& Reyna, 1998; Carneiro \& Fernandez, 2010; Neuschatz, Benoit, \& Payne, 2003; Robinson \& Roediger, 1997; Roediger \& McDermott, 1995; Stein et al., 2006; Stein \& Pergher, 2001; Toglia et al., 1999) existem evidências empíricas que apoiam os argumentos de ambas as teorias. Se, por um lado, os estudos apontam para uma relação positiva entre a força associativa reversa e as taxas de recordação e reconhecimento do distrator crítico (Deese, 1959b; McEvoy, Nelson, \& Komatsu, 1999; Roediger, Watson, et al., 2001), também não é menos verdade que, há casos em que, apesar da elevada força associativa das listas, o fenômeno não se verifica (Gallo \& Roediger, 2002). Do mesmo modo, há listas que, apesar de possuírem valores de força associativa reversa muito baixa, como é o caso de algumas das listas situacionais usadas por Cann e colaboradores (2010), geram a produção de índices elevados de recordação e reconhecimento do distrator crítico. As listas situacionais, assim classificadas de acordo com a taxonomia proposta por Wu e Barsalou (2009), caracterizam-se pelo fato das palavras que as compõem terem com o distrator crítico uma relação contextual (e.g., a lista aeroporto é constituída pelas palavras: assentos, bagagem, passaporte, pilotos, aviões, restaurantes, pista, segurança, taxi, terminal, bilhetes, viajantes).

A forte relação encontrada entre os valores de força associativa reversa das palavras apresentadas com o distrator crítico e as falsas memórias é tida como o mais claro argumento a favor da teoria de ativação e monitoramento. Para os defensores desta teoria, sendo a força associativa reversa a medida da probabilidade de cada uma das palavras apresentadas levar à produção do distrator crítico, e havendo uma correlação positiva forte entre essa variável e os índices de falsas memórias, então isso significa que o mecanismo que subjaz a esse fenômeno é um mecanismo de ativação automática do distrator crítico no momento de apresentação das palavras das listas (Deese, 1959b; McEvoy et al., 1999; Roediger, Watson, et al., 2001).

No entanto, os autores que defendem a teoria do traço difuso apresentam uma explicação diferente. Argumentam que a força associativa reversa não é mais do que uma covariável que mascara a importância que as relações semânticas entre as palavras apresentadas e o distrator crítico têm neste processo. De fato, vários estudos vieram demonstrar o elevado componente semântico das associações entre as palavras das listas usadas em tarefas DRM e os respectivos distratores críticos (Brainerd et al., 2008; Cann et al., 2010). Outros estudos (Stein \& Gomes, 2009; Stein et al., 2006) atestam a existência de uma correlação positiva entre a força associativa e outras variáveis que favorecem a memória, como a concretude das palavras que compõem as listas (Fliessbach, Weis, Klaver, Elger, \& Weber, 2006), e ressaltam a importância de controlar possíveis covariáveis no momento de selecionar as listas para os experimentos, de modo a evitar que os efeitos observados possam ser contaminados por essas mesmas covariáveis.

Por outro lado, num estudo recente de Buratto, Gomes, Prusokowski e Stein (2013), em que são apresentadas normas de associação entre palavras para 44 listas da versão brasileira do paradigma DRM, desenvolvidas por Stein e colaboradores (2006), os autores não encontraram relação entre a conectividade e a emocionalidade das listas, contrariando assim a hipótese de coesão semântica avançada em estudos anteriores (Maratos, Allan, \& Rugg, 2000). De acordo com esta hipótese, o fato das listas com conteúdo emocional gerarem taxas mais elevadas de falsas memórias se explicaria por uma maior partilha semântica entre as palavras que compõem as listas com conteúdo emocional. No estudo de Buratto e colaboradores (2013) não foi encontrada relação entre os valores de conectividade das listas e a emocionalidade (valência e arousal), quer das listas, quer dos distratores críticos. Estes resultados parecem indicar que o fato das listas emocionais gerarem mais falsas memórias não pode ser completamente explicado pela conectividade, e chamam, mais uma vez, a atenção para a necessidade de estudar melhor a natureza das relações, semânticas ou outras, entre as palavras que compõem as listas DRM.

Estes resultados vão ao encontro dos dados obtidos por McNeely, Dywan e Segalowitz (2003) num estudo em que os autores tiveram como objetivo estudar o impacto da emocionalidade no falso reconhecimento de palavras com recurso a medidas comportamentais e registros eletrofisiológicos (ERP). Num primeiro experimento, McNeely e colaboradores (2003) obtiveram achados semelhantes aos alcançados por Maratos e colaboradores (2000): as palavras com valência emocional geraram taxas superiores de acertos e de falsos alarmes em uma tarefa de reconhecimento. Relativamente aos dados de potenciais evocados, para os estímulos emocionais, registrou-se uma maior positividade nas componentes de ondas entre os 400 e os $800 \mathrm{~ms}$ nas regiões posteriores, um efeito semelhante ao efeito old/new. Uma maior positividade registrou-se também para componentes de ondas mais tardias (800 a 1,400 ms), sobretudo para o hemisfério direito, o que parece refletir um melhor processamento para informação de natureza emocional. Apesar de terem encontrado resultados semelhantes, Maratos e colaboradores (2000) atribuiram estes resultados ao fato das palavras emocio- 
nais apresentarem valores de associação semântica mais elevada. A hipótese da coesão semântica foi, no entanto, refutada por um segundo experimento realizado por McNeely e colaboradores (2003). Neste experimento, os autores usaram palavras com valência emocional negativa, palavras com elevada relação semântica e palavras neutras. Os resultados obtidos, quer nas medidas comportamentais, quer nos indicadores eletrofisiológicos, foram semelhantes aos do Experimento 1: as palavras com valência emocional negativa foram mais recordadas, originaram a produção de mais falsas memórias e a sua recordação foi acompanhada por uma maior positividade no registro eletrofisiológico comparativamente às palavras neutras, mas também às palavras com elevada associação semântica. Estes resultados parecem indicar que a maior taxa de memórias falsas gerada pelas palavras emocionais não pode ser explicada apenas por variáveis de natureza semântica, refutando, assim, a hipótese da coesão semântica avançada anteriormente por Maratos e colaboradores (2000).

$\mathrm{O}$ fato das listas com elevados valores de força associativa reversa originarem níveis de falsas memórias mais elevados do que os encontrados por S. M. Smith, Ward, Tindell, Sifonis e Wilkenfeld (2000) com listas categoriais (e.g., a lista da categoria fruta, teria como distrator crítico o exemplar mais saliente da categoria - maçã - e seria composta pelos exemplares: laranja, morango, banana, pera, pêssego, ananás, ameixa, damasco, manga, papaia, tangerina e cereja) ou listas compostas por palavras com relações semânticas do tipo situacional (Cann et al., 2010), são argumentos fortes a favor da teoria de ativação e monitoramento. Apesar de serem listas muito fortes na capacidade de extração do tema, as listas categoriais e situacionais, não raras vezes, apresentam baixos valores de força associativa reversa. Outros estudos realizados com listas de palavras associadas fonologicamente (e.g., padrão, tostão, montão, etc.), em que se verifica um efeito semelhante ao obtido com listas de associação semântica (McDermott \& Watson, 2001; Watson, Balota, \& Roediger, 2003), mostram que o caráter semântico das relações entre as palavras apresentadas e o distrator crítico não é uma condição necessária para o aparecimento das falsas memórias.

Por outro lado, a obtenção de índices de recordação e reconhecimento falsos mais elevados em tarefas que envolvem um processamento profundo (Craik \& Lochhart, 1972), por oposição a tarefas de processamento não profundo (Stein \& Pergher, 2001; Toglia et al., 1999), parecem evidenciar o envolvimento de variáveis de natureza semântica. Estes resultados parecem corroborar a hipótese das falsas memórias serem o resultado da formação de um tema aglutinador do significado das palavras que compõem as listas, uma vez que o processamento profundo favorece a possibilidade de extração do tema da lista, enquanto em tarefas de processamento superficial, a haver ativação do distrator crítico, esta será automática. Do mesmo modo, o fato de vários estudos (Toglia et al., 1999) mostrarem a persistência das memórias falsas, por comparação com as verdadeiras, ao longo do tempo, parece indicar que estas são resultado da extração de um tema geral que tende a perdurar no tempo.

A favor da teoria do traço difuso parecem estar também resultados de vários estudos (Toglia, Hinman, Dayton, \& Catalano, 1997; Toglia et al., 1999) que mostram a importância do formato de apresentação das palavras nas listas (bloco vs. aleatório). No formato de apresentação em bloco, as palavras são apresentadas lista-a-lista, enquanto no formato aleatório são apresentadas todas as palavras de várias listas de forma aleatória. Neste caso, e apesar da força associativa se manter constante, o que deveria levar a níveis de ativação do distrator crítico semelhantes, a apresentação das palavras em bloco favorece o aparecimento das falsas memórias, o que pode ser explicado pelo fato deste formato de apresentação favorecer a extração do tema das listas. No mesmo sentido, apontam resultados de estudos que revelam que, quando as palavras associadas são apresentadas por ordem de associação decrescente com o distrator crítico, se obtêm mais falsos alarmes para os distratores críticos do que quando a ordem é crescente ou aleatória (Brainerd et al., 2001). Ou seja, para a formação do distrator crítico, a ordem de apresentação das palavras não é um aspecto desprezível. $\mathrm{O}$ fato dos participantes processarem em primeiro lugar um associado mais forte, seguido de palavras com um menor grau de associação, pode promover a recordação do distrator crítico, na medida em que pode favorecer a extração do tema das listas (Brainerd et al., 2001). Neste sentido apontam também alguns trabalhos recentes em que o objetivo foi o de estudar a capacidade de identificação dos temas das listas, e que mostraram que a taxa de identificação do tema era maior quando os associados mais fortes eram as primeiras palavras das listas (Albuquerque \& Resende, 2011).

Assim, para a compreensão integral deste fenômeno, parece necessário esclarecer o tipo de relações que está na base das associações entre as palavras que compõem as listas e os respectivos distratores críticos. Mas, além disso, importa também compreender o papel de outras variáveis relativas à estrutura das listas. Se a ativação do distrator crítico, ou a extração do tema das listas, dependesse apenas da força associativa reversa ou do tipo de relações semânticas entre os conceitos, então, não se esperariam variações em função da ordem de apresentação das palavras nas listas (Brainerd et al., 2001) ou da sua apresentação aleatória ou em bloco (Toglia et al., 1997).

Das evidências empíricas existentes até ao momento, parece poder concluir-se que, por um lado, os processos que levam ao aparecimento de falsas memórias no paradigma DRM, os mecanismos de ativação, são de ordem diversa e que, por outro lado, para uma compreensão abrangente do fenômeno, há que se levar em conta outros processos que contribuem para a inibição ou eliminação das falsas memórias, os mecanismos de monitoramento ou controle. 


\section{Mecanismos de Controle ou Monitoramento}

Consideram-se mecanismos de controle ou monitoramento, os processos através dos quais, os participantes, numa tarefa de memória, inibem o surgimento de uma falsa memória. Entre estes, contam-se processos de revisão e de decisão que, ao permitirem identificar a fonte da ativação do distrator crítico, conduzem à sua não recordação ou à sua correta rejeição (Gallo, 2010).

De acordo com a teoria de ativação e monitoramento, o controle das falsas memórias dá-se, sobretudo, através de um mecanismo de monitoramento da fonte da ativação. Tendo ocorrido a ativação do distrator crítico na fase de estudo, ou mesmo na fase de teste, a sua recordação ou reconhecimento podem não ocorrer, caso os participantes sejam capazes de identificar corretamente a fonte (interna) dessa ativação. Na prática, isso ocorreria quando os participantes fossem capazes de discernir entre as palavras que efetivamente foram apresentadas e aquelas em que apenas pensaram (Roediger \& McDermott, 2000a, 2000b). O mecanismo de controle previsto pela teoria de ativação e monitoramento deriva da noção de monitoramento da realidade, inicialmente proposta por Johnson (1988) como dizendo respeito à capacidade para distinguir entre eventos reais ou imaginados. De acordo com este modelo, os erros seriam resultado da confusão entre memórias para estes dois tipos de eventos. Mais tarde, Johnson e colaboradores (1993) propuseram a noção de monitoramento da fonte, de acordo com a qual a maioria dos erros poderia ser explicada por confusões relativas à fonte dos acontecimentos. Isso significa que uma falsa memória poderá ser resultado de uma atribuição externa (e.g., ter visto uma palavra) a uma memória que, na verdade, tenha tido origem interna (e.g., ter pensado numa palavra), ou de uma atribuição errada à fonte da informação, podendo esta ser de origem interna (e.g., pensar em algo e julgar tê-lo dito) ou externa (e.g., ouvir na rádio e julgar ter visto na televisão).

Segundo a teoria do traço difuso, na fase de estudo, junto com a codificação do tema das listas, dá-se o processamento das características perceptivas das palavras apresentadas (e.g., tamanho ou número de letras que compõem uma palavra), dando origem à formação de um traço mnemônico denominado pelos autores desta teoria por traço literal. A posterior recordação destes aspectos, e a consequente recordação e reconhecimento das palavras apresentadas, constituiria uma forma de controle das falsas memórias, uma vez que se trata de características específicas dos itens apresentados. Esta estratégia, designada de recordar-para-rejeitar, é, segundo os autores que defendem a teoria do traço difuso, a principal forma de evitar a recordação e reconhecimento do distrator crítico (Brainerd et al., 2001; Reyna, 2000).

Em qualquer dos casos, considera-se que a identificação correta, e posterior recordação e reconhecimento das palavras apresentadas, constitui uma forma de monitoramento das falsas memórias. De fato, inúmeros estudos corroboram a ideia de que existe uma correlação negativa entre a recordação correta e a recordação do distrator crítico (Deese, 1959a, 1959b; Gallo \& Roediger, 2002; McEvoy et al., 1999; Roediger, Watson, et al., 2001). Baseado nesta ideia, Gallo $(2004,2006,2010)$ apresentou uma tipologia de processos de monitoramento. De acordo com esta tipologia, o monitoramento pode ocorrer por duas vias distintas: diagnóstica ou desqualificante.

Diz-se que há monitoramento diagnóstico nas situações em que a expectativa da recordação de determinados detalhes associados a um evento é de tal forma elevada que a ausência de um traço mnemônico correspondente leva os indivíduos a concluir pela inexistência do evento (e.g., "se a palavra bárbara fizesse parte da lista, eu recordar-me-ia, porque esse é meu nome preferido!"'). Neste caso, a rejeição da falsa memória relativa a um eventual acontecimento ou palavra é baseada na ausência de informação relativa a esse mesmo acontecimento ou palavra (Gallo, 2004, 2010).

No caso das tarefas DRM, o monitoramento diagnóstico depende, fundamentalmente, das características do distrator crítico e da medida em que ele é distintivo. A heurística da distintividade constitui, assim, um mecanismo importante de rejeição das falsas memórias (Schacter, Israel, \& Racine, 1999), e uma boa explicação para os resultados encontrados em vários estudos, designadamente estudos que se centram no papel que as características da palavra crítica desempenham neste fenômeno. De acordo com alguns destes estudos, quando o distrator crítico tem características muito distintivas em relação às palavras das listas (e.g., no número de letras), a probabilidade de ele ser falsamente recordado ou reconhecido é menor (Madigan $\&$ Neuse, 2004; Roediger, Watson, et al., 2001).

Embora o monitoramento diagnóstico diga respeito, sobretudo, a uma decisão primária, por ser baseada na falta de informação relativa a um evento, e não na informação recordada acerca desse mesmo evento (Gallo, 2010), alguns autores defendem que este tipo de monitoramento também pode ser facilitado pela correta identificação das palavras apresentadas, na medida em que isso poderá tornar mais saliente a ausência de um dado item (Gallo, 2004). Neste sentido apontam alguns estudos (Cleary \& Greene, 2002; Gallo, McDermott, Percer, \& Roediger, 2001; Israel \& Schacter, 1997; R. E. Smith \& Hunt, 1998) nos quais se manipulou a modalidade de apresentação dos estímulos, tendo-se constatado que modalidades que permitem a codificação de informação mais distintiva acerca dos itens apresentados conduzem a taxas de falsas memórias mais baixas. Portanto, quando são apresentadas imagens (estímulos mais ricos em detalhes perceptivos) a taxa de reconhecimento falso diminui, o mesmo acontecendo quando se compara a modalidade visual com a apresentação auditiva das palavras. Outros estudos mostram também uma vantagem da apresentação tátil dos estímulos sobre a auditiva (Nabeta \& Kawahara, 2006). Saliente-se ainda que, em todos estes estudos, junto com uma diminuição dos índices de falsas memórias, se registrou um aumento 
da recordação e do reconhecimento corretos, o que poderá apontar para o envolvimento de mecanismos de monitoramento desqualificante.

O monitoramento diagnóstico só é viável quando as características do estímulo o destacam, isto é, quando uma palavra é saliente ou muito distintiva. Acontece que, na maior parte das vezes, a informação a inibir não possui essas características. No caso das listas DRM, os distratores críticos associados são, maioritariamente, palavras comuns para os participantes. Nestes casos, o monitoramento terá de ser realizado por desqualificação, ou seja, com base na informação que os participantes conseguem recordar das listas (Gallo, 2010).

O monitoramento desqualificante pode ocorrer de duas formas distintas. Uma das possibilidades passa pela recordação de todas as palavras de uma lista e, sabendo o número de itens que a compõem, por exclusão de partes, pela consequente rejeição do distrator crítico. Outra possibilidade de desqualificação envolve a identificação do próprio distrator crítico das listas como sendo uma palavra à qual todas as apresentadas estão associadas, mas que não foi apresentada. Neste caso, estamos perante uma estratégia conhecida como identificar-para-rejeitar (Gallo, 2004, 2010).

O fato da identificação do distrator crítico das listas poder constituir-se como uma forma de monitoramento, está bem documentado por vários estudos em que é manipulado o grau de identificabilidade do tema das listas (Albuquerque \& Resende, 2011; Carneiro, Fernandez, \& Dias, 2009). De acordo com os resultados obtidos nestes estudos, as listas de palavras em que o tema é fácil de identificar apresentam taxas mais baixas de produção de falsas memórias comparativamente a listas em que o tema é de difícil identificação. Do mesmo modo, quando os participantes são avisados da existência de um distrator crítico, verifica-se uma redução na sua recordação e reconhecimento, porque aumenta a probabilidade dos participantes recorrerem a esta estratégia como forma de controle das falsas memórias (Carneiro \& Fernandez, 2010; Neuschatz et al., 2003).

Além da identificação do distrator crítico, o monitoramento desqualificante, também pode resultar da correta identificação das palavras das listas. Neste caso, estamos perante uma estratégia denominada de recordar-para-rejeitar. $\mathrm{O}$ caso mais extremo verifica-se quando se dá a correta recordação de todas as palavras de uma lista, e, por exclusão de partes, a desqualificação do distrator crítico. Este poderá ser o processo que se verifica quando o número de palavras das listas é relativamente baixo, mas torna-se menos fácil quando as listas são compostas por maior número de associados, já que, neste caso, a recordação exaustiva de todos os itens se torna uma tarefa mais difícil. De fato, em alguns estudos (Robinson \& Roediger, 1997) em que se manipulou o número de palavras das listas, verificou-se que a probabilidade de ocorrência de falsas memórias em listas mais curtas (e.g., 3, 6 ou 9 associados) era mais baixa. No entanto, neste caso, o resultado também pode ser explicado pelo fato de, nestas listas, o nível de ativação do distrator crítico ser menor, já que há menos palavras a contribuírem para a ativação do distrator crítico. Porém, há estudos que mostram que, quando todas as palavras de uma lista são recordadas, a probabilidade de recordar o distrator crítico decresce (Gallo, 2004). Além disso, a existência de uma correlação negativa entre memórias corretas e falsas memórias, descrita em muitos estudos (Deese, 1959b; Gallo \& Roediger, 2002; McEvoy et al., 1999; Roediger, Watson, et al., 2001), também pode evidenciar que a correta identificação das palavras apresentadas constitui uma forma de monitoramento das falsas memórias.

Assim, parecem existir evidências de que o controle das falsas memórias pode ocorrer de várias formas, e que, não se tratando de processos mutuamente exclusivos, eles podem ocorrer simultaneamente ou interagir em tarefas de memória. Se é verdade que a correta identificação das propriedades dos itens das listas pode levar à desqualificação do distrator crítico (como defendem os autores que preconizam a teoria do traço difuso), também é um fato que a identificação desse mesmo distrator crítico, junto com a correta identificação da sua fonte de ativação (como argumentam os autores que defendem a teoria de ativação e monitoramento), constitui um importante mecanismo de controle das falsas memórias. Independentemente dos processos que as duas teorias consideram estar na base do aparecimento das falsas memórias, ambas contemplam mecanismos do controle. Estes mecanismos pretendem explicar os motivos pelos quais listas construídas de forma semelhante conduzem as taxas de recordação e reconhecimento do distrator crítico tão díspares, mesmo quando o procedimento experimental é o mesmo.

\section{Considerações Finais}

Com este artigo pretendeu-se refletir sobre os mecanismos explicativos da produção de falsas memórias no paradigma DRM. A partir da revisão da literatura efetuada é possível concluir que, apesar do vasto número de estudos realizados até ao momento, os mecanismos que levam à ocorrência de memórias falsas no paradigma DRM não estão ainda completamente esclarecidos.

Uma questão central que divide as duas grandes teorias explicativas do fenômeno é a de saber se as falsas memórias (neste caso, a recordação ou reconhecimento do item crítico) resultam de um processo associativo que ocorre de forma automática, no momento da codificação e/ou da recuperação das palavras apresentadas, ou se derivam da extração do tema ou do significado geral das listas. No que respeita aos mecanismos de ativação, embora as perspectivas das duas teorias apresentadas sejam diferentes, elas não são inconciliáveis. Para os autores que defendem a teoria de ativação e monitoramento, para que haja uma falsa memória, é necessário que ocorra a ativação de uma representação mnemônica de um conceito (não apresentado) em consequência da apresentação de um estímulo 
que tem com aquele algum tipo de relação, seja esta de natureza semântica, situacional, fonológica, categorial ou outra (Gallo \& Roediger, 2002; Roediger, Balota, et al., 2001; Roediger \& McDermott, 1995, 2000a, 2000b). Na da teoria do traço difuso, assume-se que este é um processo eminentemente semântico, que decorre da formação de um traço mnemônico de essência, e que, no caso do paradigma DRM, se consubstancia na extração do tema das listas, sendo que este terá com as palavras apresentadas algum tipo de relação semântica (Brainerd \& Reyna, 2005; Reyna \& Brainerd, 1995).

Tendo em conta a evidência empírica apresentada, parece claro que a associação semântica entre as palavras de uma lista e o distrator crítico é uma variável muito importante, no entanto, esta não é suficiente para dar conta de todos os resultados encontrados. Uma das dificuldades em determinar com precisão a natureza das associações que levam ao aparecimento das memórias falsas é o fato de existir uma elevada sobreposição entre os diferentes tipos de relações associativas. Vários estudos (Brainerd et al., 2008; Cann et al., 2010; McRae et al., 2012) mostram que grande parte das listas DRM apresentam uma elevada associação semântica com os respectivos distratores cíticos, e alguns autores (Stein \& Gomes, 2009; Stein et al., 2006) chamam a atenção para a importância de controlar covariáveis que possam interferir na ocorrência das falsas memórias, como a emocionalidade ou a concretude.

Na tentativa de clarificar o impacto das relações semânticas e associativas, Carneiro, Garcia-Marques, Fernandez e Albuquerque (2014), usaram recentemente listas associativas (listas com elevada força associativa reversa e baixa capacidade de extração do tema) e listas temáticas (listas fortes na capacidade de extração do tema e com baixa força associativa). Os valores de falso reconhecimento foram mais elevados para as listas associativas, confirmando, uma vez mais, a importância que a ativação automática tem neste processo. No entanto, registraram-se também valores consideráveis (na ordem dos 50\%) de falso reconhecimento para as listas temáticas, mostrando que a extração do tema, e, portanto, as relações semânticas, desempenha um papel que não pode ser desprezado. $\mathrm{O}$ fato dos autores terem manipulado de forma independente as duas variáveis tidas por ambas as teorias como sendo fundamentais, e terem obtido valores consideráveis de falso reconhecimento também para listas com baixa força associativa, parece, confirmar a necessidade de adotar uma abordagem teórica que considere, quer a ativação automática, como propõe a teoria de ativação e monitoramento, quer a formação de um traço mnemônico de essência, como defendem os autores da teoria do traço difuso.

Se, no que toca aos mecanismos de ativação, as visões de ambas as teorias podem ser conciliadas, relativamente aos mecanismos de monitoramento e controle podemos dizer que as suas perspectivas se complementam. Prova disso é a tipologia apresentada por Gallo (2004, 2006, 2010), que contempla dois tipos de estratégias de monitoramento: diagnóstica e desqualificante. Se há situações em que o sucesso do monitoramento é devido à distintividade das características do distrator crítico, sendo que, numa tarefa de reconhecimento a sua rejeição se deve à ausência de um traço mnemônico literal para esse estímulo, como previsto pela teoria do traço difuso (Brainerd \& Reyna, 2005; Reyna \& Brainerd, 1995), na maior parte dos casos, o monitoramento tem de ser feito por desqualificação, ou seja, com base na informação apresentada e/ ou recordada. A este nível, parece haver evidência que a identificação do tema das listas (identificar-para-rejeitar) é uma estratégia comumente usada pelos participantes para identificar uma palavra como não tendo sido apresentada, seja esta resultado de avisos explicitamente feitos pelos experimentadores (Neuschatz et al., 2003), ou adotada voluntariamente pelos participantes, nos casos em que a identificação do tema das listas é muito fácil (Carneiro \& Fernandez, 2010).

Num estudo recente, Carneiro e Fernandez (2013) pediram aos participantes para, no final de uma tarefa de recordação, dizerem quais as estratégias de memorização que tinham utilizado. A identificação do tema das listas foi referida por cerca de um terço dos participantes e, destes, mais de metade, referiam ter usado esta estratégia como forma de inibir as falsas memórias. Estes dados parecem suportar a hipótese da formação de um traço mnemônico de essência no momento da codificação, como defendem os autores do traço difuso (Brainerd \& Reyna, 2005; Reyna \& Brainerd, 1995), no entanto, também vão ao encontro de ideia de que, para haver monitoramento, os participantes devem identificar corretamente a fonte de ativação de um estímulo, tal como argumentam os autores da teoria de ativação e monitoramento (Gallo \& Roediger, 2002; Roediger, Balota, et al., 2001; Roediger \& McDermott, 1995, 2000a, 2000b). Ou seja, a correta identificação do tema das listas, não garante, por si só, a inibição da falsa memória. Para tal, é necessário que os participantes, no momento da recuperação, identifiquem corretamente a fonte dessa informação. Ou seja sejam capazes de perceber que pensaram nessa palavra (porque estava associada a todas as palavras da lista), mas que ela não fazia parte da lista apresentada.

Assim, tal como para os mecanismos de ativação, também para os mecanismos de controle, parece ser necessário adotar uma posição conciliadora entre as duas teorias. Deste modo, propõe-se a adoção de uma abordagem teórica que integre as perspectivas de ambas as teorias, contribuindo para uma explicação mais completa deste fenômeno.

\section{Referências}

Albuquerque, P. B., \& Resende, A. (2011, August). The relation between theme identifiability and false memories: Exacerbating positions... reducing the effect. Poster presented at International Conference on Memory - ICOM 5, York, England.

Brainerd, C. J., \& Reyna, V. F. (1998). When things that were never experienced are easier to "remember" than things that were. Psychological Science, 9, 484-489. doi:10.1111/14679280.00089 
Brainerd, C. J., \& Reyna, V. F. (2005). The science of false memory. New York: Oxford University Press. doi:10.1093/ acprof:oso/9780195154054.001.0001

Brainerd, C. J., Wright, R., Reyna, V. F., \& Mojardin, A. H. (2001). Conjoint recognition and phantom recollection. Journal of Experimental Psychology: Learning, Memory, \& Cognition, 27, 307-327. doi:10.1037/0278-7393.27.2.307

Brainerd, C. J., Yang, Y., Reyna, V. F., Howe, M. L., \& Mills, B. A. (2008). Semantic processing in "associative" false memory. Psychonomic Bulletin \& Review, 15, 1035-1053. doi:10.3758/PBR.15.6.1035

Buratto, L. G., Gomes, C. F. A., Prusokowski, T. S., \& Stein, L. M. (2013). Inter-item associations for the Brazilian version of the Deese/Roediger-McDermott paradigm. Psicologia: Reflexão e Crítica, 26(2), 367-375. doi:10.1590/S010279722013000200017

Cann, D. R., McRae, K., \& Katz, A. N. (2010). False recall in the Deese-Roediger-McDermott paradigm: The roles of gist and associative strength. Quarterly Journal of Experimental Psychology, 64(8), 1515-1542. doi:10.1080/17470218.201 1.560272

Carneiro, P., \& Fernandez, A. (2010). Age differences in the rejection of false memories: The effects of giving warning instructions and slowing the presentation rate. Journal of Experimental Child Psychology, 105, 81-97. doi:10.1016/j. jecp.2009.09.004

Carneiro, P., \& Fernandez, A. (2013). Retrieval dynamics in false recall: Revelations from identifiability manipulations. Psychonomic Bulletin \& Review, 20, 488-495. doi:10.3758/ s13423-012-0361-4

Carneiro, P., Fernandez, A., \& Dias, A. R. (2009). The influence of theme identifiability on false memories: Evidence for age-dependent opposite effects. Memory \& Cognition, 37, 115-129. doi:10.3758/MC.37.2.115

Carneiro, P., Garcia-Marques, L., Fernandez, A., \& Albuquerque, P. (2014). Both associative activation and thematic extraction count, but thematic false memories are more easily rejected. Memory, 22(8), 1024-1040. doi:10.1080/09658211.2013.8 64680

Cleary, A. M., \& Greene, R. L. (2002). Paradoxical effects of presentation modality on false memory. Memory, 10(1), 5561. doi:10.1080/09658210143000236

Collins, A. M., \& Loftus, E. F. (1975). A spreading-activation Theory of Semantic Processing. Psychological Review, 82(6), 407-428. doi:10.1037/0033-295X.82.6.407

Collins, A. M., \& Quillian, M. R. (1969). Retrieval time from semantic memory. Journal of Verbal Learning and Verbal Behavior, 8, 240-248. doi:10.1016/S0022-5371(69)80069-1

Collins, A. M., \& Quillian, M. R. (1970). Facilitating retrieval from semantic memory: The effect of repeating part of an inference. Acta Psychologica, 33, 304-314. doi:10.1016/00016918(70)90142-3

Craik, F. I. M., \& Lockhart, R. S. (1972). Levels of processing: A framework for memory research. Journal of Verbal Learning and Verbal Behavior, 11, 671-684. doi:10.1037/h0084237

Cramer, R. (1970). Semantic generalization: IAR locus and instructions. Journal of Experimental Psychology, 83, 266-273. doi: $10.1037 / \mathrm{h} 0028534$

Deese, J. (1959a). Influence of inter-item associative strength upon immediate free recall. Psychological Reports, 5, 305312. doi:10.2466/PR0.5.3.305-312

Deese, J. (1959b). On the prediction of occurrence of particular verbal intrusions in immediate recall. Journal of Experimental Psychology, 58, 17-22. doi:10.1037/h0046671
Ebbinghaus, H. (1964). Memory: A contribution to experimental psychology. New York: Dover. (Original work published 1885)

Fliessbach, K., Weis, S., Klaver, P., Elger, C. E., \& Weber, B. (2006). The effect of word concreteness on recognition memory. NeuroImage, 32, 1413-1421. doi:10.1016/j.neuroimage.2006.06.007

Gallo, D. A. (2004). Using recall to reduce false recognition: Diagnostic and disqualifying monitoring. Journal of Experimental Psychology: Learning, Memory, \& Cognition, 30, 120-128. doi:10.1037/0278-7393.30.1.120

Gallo, D. A. (2006). Associative illusions of memory: False memory research in DRM and related tasks. New York: Psychology Press.

Gallo, D. A. (2010). False memories and fantastic beliefs: 15 years of the DRM illusion. Memory \& Cognition, 38(7), 833 848. doi:10.3758/MC.38.7.833

Gallo, D. A., McDermott, K. B., Percer, J. M., \& Roediger, H. L. (2001). Modality effects in false recall and false recognition. Journal of Experimental Psychology: Learning, Memory, \& Cognition, 27, 339-353. doi:10.1037/0278-7393.27.2.339

Gallo, D. A., \& Roediger, H. L. (2002). Variability among word lists in eliciting memory illusions: Evidence for associative activation and monitoring. Journal of Memory and Language, 47, 469-497. doi:10.1016/S0749-596X(02)00013-X

Israel, L., \& Schacter, D. L. (1997). Pictorial encoding reduces false recognition of semantic associates. Psychonomic Bulletin \& Review, 4, 577-581. doi:10.3758/BF03214352

Johnson, M. K. (1988). Reality monitoring: An experimental phenomenological approach. Journal of Experimental Psychology: General, 117(4), 390-394. doi:10.1037/00963445.117.4.390

Johnson, M. K., Hashtroudi, S., \& Lindsay, D. S. (1993). Source monitoring. Psychological Bulletin, 114(1), 3-28. doi:10.1037/0033-2909.114.1.3

Kirkpatrick, E. A. (1894). An experimental study of memory. Psychological Review, 1(6), 602-609.

Madigan, S., \& Neuse, J. (2004). False recognition and word length: A reanalysis of Roediger, Watson, McDermott, and Gallo (2001) and some new data. Psychonomic Bulletin \& Review, 11, 567-573. doi:10.3758/BF03196612

Maratos, E. J., Allan, K., \& Rugg, M. D. (2000). Recognition memory for emotionally negative and neutral words: An ERP study. Neuropsychologia, 38, 1452-1465. doi:10.1016/ S0028-3932(00)00061-0

McEvoy, C. L., Nelson, D. L., \& Komatsu, T. (1999). What is the connection between true and false memories? The differential roles of inter-item associations in recall and recognition. Journal of Experimental Psychology: Learning, Memory, and Cognition, 25(5), 1177-1194. doi:10.1037/0278-7393.25.5.1177

McDermott, K. B., \& Watson, J. M. (2001). The rise and fall of false recall: The impact of presentation duration. Journal of Memory \& Language, 45, 160-176. doi:10.1006/ jmla.2000.2771

McNeely, H. E., Dywan, J., \& Segalowitz, S. J. (2003). ERP indices of emotionality and semantic cohesiveness during recognition judgments. Psychophysiology, 41(1), 117-129. doi:10.1111/j.1469-8986.2003.00137.x

McRae, K., Khalkhali, S., \& Hare, M. (2012). Semantic and associative relations in adolescents and young adults: Examining a tenuous dichotomy. In V. F. Reyna, S. Chapman, M. Dougherty, \& J. Confrey (Eds.), The adolescent brain: Learning, reasoning, and decision making (pp. 39-66). 
Washington, DC: American Psychological Association. doi:10.1037/13493-002

Meade, M. L., Watson, J. M., Balota, D. A., \& Roediger H. L. (2007). The roles of spreading activation and retrieval mode in producing false recognition in the DRM paradigm. Journal of Memory and Language, 56, 305-320. doi:10.1016/j. jml.2006.07.007

Nabeta, T., \& Kawahara, J. (2006). Reduction of false recognition through haptic presentation of objects. European Journal of Cognitive Psychology, 18(6), 801-812. doi:10.1080/09541440500334482

Neuschatz, J. S., Benoit, G., \& Payne, D. G. (2003). Effective warnings in the Deese-Roediger-McDermott false-memory paradigm: The role of identifiability. Journal of Experimental Psychology: Learning, Memory, \& Cognition, 29, 35-41. doi:10.1037/0278-7393.29.1.35

Pimentel, E., \& Albuquerque, P. B. (2013). Effect of divided attention on the production of false memories in the DRM paradigm: A study of dichotic listening and shadowing. Psicológica, 34, 285-298.

Quillian, M. R. (1967). Word concepts: A theory and simulation of some basic semantic capabilities. Behavioral Science, 12, 410-430. doi:10.1002/bs.3830120511

Reyna, V. F. (2000). Fuzzy-trace theory and source monitoring: An evaluation of theory and false-memory data. Learning and Individual Differences, 12, 163-175. doi:10.1016/S10416080(01)00034-6

Reyna, V. F., \& Brainerd, C. J. (1995). Fuzzy-trace theory: An interim synthesis. Learning \& Individual Differences, 7, 1-75. doi:10.1016/1041-6080(95)90031-4

Rips, L. J., Shoben, E. J., \& Smith, E. E. (1973). Semantic distance and the verification of semantic relations. Journal of Verbal Learning and Verbal Behavior, 12, 1-20. doi:10.1016/ S0022-5371(73)80056-8

Robinson, K. J., \& Roediger H. L. (1997). Associative processes in false recall and false recognition. Psychological Science, 8(3), 231-237. doi:10.1111/j.1467-9280.1997.tb00417.x

Roediger, H. L., Balota, D. A., \& Watson, J. M. (2001). Spreading activation and the arousal of false memories. In H. L. Roediger, J. S. Nairne, I. Neath, \& A. M. Suprenant (Eds.), The nature of remembering: Essays in honor of Robert $G$. Crowder (pp. 95-115). Washington, DC: American Psychological Association Press. doi:10.1037/10394-006

Roediger, H. L., \& McDermott, K. B. (1995). Creating false memories: Remembering words not presented in lists. Journal of Experimental Psychology: Learning, Memory, and Cognition, 21(4), 803-814. doi:10.1037/0278-7393.21.4.803

Roediger, H. L., \& McDermott, K. B. (2000a). Distortions of memory. In E. Tulving \& F. I. M. Craik (Eds.), The Oxford handbook of memory (pp. 149-162). New York: Oxford University Press.

Roediger, H. L., \& McDermott, K. B. (2000b). Tricks of memory. Current Directions in Psychological Science, 9, 123-127. doi:10.1111/1467-8721.00075

Roediger, H. L., Watson, J. M., McDermott, K. B., \& Gallo, D. A. (2001). Factors that determine false recall: A multiple regression analysis. Psychonomic Bulletin \& Review, 8(3), 385-407. doi:10.3758/BF03196177

Schacter, D. L., Israel, L., \& Racine, C. (1999). Suppressing false recognition in younger and older adults: The distinctiveness heuristic. Journal of Memory and Language, 40, 1-24. doi:10.1006/jmla.1998.2611
Smith, E. E., Shoben, E. J., \& Rips, L. J. (1974). Structure and process in semantic memory: A featural model for semantic decisions. Psychological Review, 81, 214-241. doi:10.1037/ h0036351

Smith, R. E., \& Hunt, R. R. (1998). Presentation modality affects false memory. Psychonomic Bulletin \& Review, 5, 710-715. doi:10.3758/BF03208850

Smith, S. M., Ward, T. B., Tindell, D. R., Sifonis, C. M., \& Wilkenfeld, M. J. (2000). Category structure and created memories. Memory and Cognition, 28(3), 386-395. doi:10.3758/BF03198554

Stein, L. M., \& Gomes, C. F. A. (2009). Normas brasileiras para listas de palavras associadas: Associação semântica, concretude, frequência e emocionalidade. Psicologia: Teoria e Pesquisa, 25(4), 537-546. doi:10.1590/S0102-37722009000400009

Stein, L. M., Feix, L. F., \& Rohenkohl, G. (2006). Avanços metodológicos no estudo das falsas memórias: Construção e normatização do procedimento de palavras associadas. Psicologia: Reflexão e Crítica, 19(2), 166-176. doi:10.1590/ S0102-79722006000200002

Stein, L. M., \& Pergher, G. K. (2001). Criando falsas memórias em adultos por meio de palavras associadas. Psicologia: Reflexão e Crítica, 14(2), 353-366. doi:10.1590/S010279722001000200010

Toglia, M. P., Hinman, P. J., Dayton, B. S., \& Catalano, J. F. (1997). The blocked-random effect in pictures and words. Perceptual and Motor Skills, 8, 976-978. doi:10.2466/ pms.1997.84.3.976

Toglia, M. P., Neuschatz, J. S., \& Goodwin, K. A. (1999). Recall accuracy and illusory memories: When more is less. Memory, 7(2), 233-256. doi:10.1080/741944069

Tulving, E. (1985). How many memory systems are there? American Psychologist, 40, 385-398. doi:10.1037/0003066X.40.4.385

Underwood, B. J. (1965). False recognition produced by implicit verbal responses. Journal of Experimental Psychology, 70, 122-129. doi:10.1037/h0022014

Watson, J. M., Balota, D. A., \& Roediger, H. L. (2003). Creating false memories with hybrid lists of semantic and phonological associates: Over-additive false memories produced by converging associative networks. Journal of Memory \& Language, 49, 95-118. doi:10.1016/S0749-596X(03)00019-6

Wu, L. L., \& Barsalou, L. W. (2009). Perceptual simulation in conceptual combination: Evidence from property generation. Acta Psychologica, 132(2), 173-189. doi:10.1016/j. actpsy.2009.02.002 